\title{
Artificial Intelligence - the new Revolutionary Evolution
}

\section{Recent advances in Artificial Intelligence (Al) have paved the way for exciting applications, which are now playing important roles in everyday life ranging from language translation and image processing to recommender systems and autonomous driving. Most applica- tions are based on machine learning, which achieved great successes due to increasingly available computational resources and data. Applying Al to diverse areas is appealing due to its self-organizing and self-learning nature, which enables generic ways of solving prob- lems with minimal effort on specifications. The current trend of applied Al offers numerous opportunities to contribute within areas of research, theory, technology, and application.}

\section{Overview - What makes a system intelligent?}

The term intelligence is generally not well defined and understood since it is a hot topic for research in various areas like biology, psychology, and neuro sciences itself. Artificial intelligence exploits the knowledge of these areas to create machines and programs which are able to solve problems in an intelligent and adaptive manner with only little problem specific knowledge than the problem formulation itself. The main components of intelligent systems are a Learning component, a Thinking, and an Acting component:

The Learning component which is usually implemented via Machine Learning methods is either trained with a lot of available data to evaluate states and moves based on Supervised Learning or Unsupervised Learning or with generated data, which is obtained automatically by using trial-error approaches like Reinforcement Learning. Reinforcement Learning has become a popular field in industry and research due to its self-adapting nature for intelligent systems. In Reinforcement Learning an autonomous entity or agent trains itself to achieve a given goal by interacting with the environment similarly to living beings. While there is no explicit guidance to achieve that goal, the agent gets numeric feedback which is known as reward signal. The reward itself is inspired by conditioning from the field of psychology. The agent has to adapt its behavior to maximize the long term expectation of the cumulative reward. In a game the goal would be to win against a strong opponent, or in navigation the goal could be to reach a target destination within given constraints.

The Thinking component involves explicit reasoning about future actions and events. In a game, different future scenarios can be considered to decide on the next move that maximizes the probability of winning, while in a navigation task, careful routing and online planning would be required to safely move an autonomous entity like a car to a desired destination without unneces- sary detours, costs, or accidents. Technically, artificial thinking is realized by search or optimization techniques like tree search (e.g., Monte Carlo Tree Search or Alpha-Beta Search), meta-heuristic optimization (e.g., Evolutionary Computation or Simulated Annealing) or routing algorithms (e.g., Dijkstra or A* Search). A model of the environment like a simulator, a map or simply the rules of the games are required for effective search. In situations, where models are not available, Machine Learning could be used to bridge the gap between Learning and Thinking. An example for this is Model-based Reinforcement Learning, which additionally learns a model of the environment, while adapting its strategy to it. Thus, Model-based Reinforcement Learning can be considered as a combination of Learning and Thinking. It closely resembles real intelligent behavior.

The Acting component makes decisions based on information provided by the Learning and the Thinking components. While Learning and Thinking are required to extract useful information from data, simulations, or other kinds of reasoning and optimization, the Acting component bears the "responsibility" of the final decisions made. The Acting component has to consider all the information either learned, thought, or provided by third parties like the engineers, the customers, other intelligent systems, etc. to make a decision which can be considered as "intelligent" by the outside world. Acting involves many aspects of different disciplines ranging from social sciences and psychology, to biology and neuro science. An important aspect of Acting is the consideration of other intelligent beings like living beings (e.g., humans) or other artificially intelligent systems. Such situations are very challenging due to each system trying to achieve its own goal (which might be in conflict to each other) without harming the global world. Acting requires a lot of responsibility, attention, and transparency to be implemented in a meaningful way, to be beneficial for industries and everyday life. 


\section{Current Challenges and Trends}

Today many of the basic techniques mentioned before are now used in various fields to realize features like Image Processing to sharpen blurry images or to detect objects at different orientations and scales, or Pattern Recognition to identify human behavior and to classify customers in order to improve the service or user experience. The extracted knowledge is often used by Recommender Systems to provide suitable advertisement to the user. Predictive Maintainance should support operative tasks by proactively inform the status to people in charge to avoid outtakes and to optimize processes. Industry 4.0 is an upcoming trend where AI methods are used to create self-organizing systems, which are able to produce individual products with only little handcrafting of manufacturing processes. Autonomous driving is one of the long standing visions of using $\mathrm{AI}$ in the real world, by using powerful learning and thinking systems to create self-adaptive vehicles which can safely transport people and objects to their target destinations. Language Processing is an important field to bridge the gap between different human cultures but also between human and machine especially in education, where robots are meant to support teachers and students in their daily tasks. Another upcoming field is AI in Healthcare, where mostly Machine Learning is used to make diagnosis and to evaluate XRay images to make more reliable decisions than humans, which can save lives. A really appealing field is using AI for Social Good. That is to use Learning, Thinking, and Acting approaches to combat common threats of nature or humanity. One example is to catch poachers or to find snares in order to protect wildlife. Recent approaches often use Game Theoretic approaches which can be classified as Acting method.

Although there are many active fields about AI, there are still many challenges to be addressed: while most successes are based on Machine Learning, which itself is enabled by the availability of computing power, scaling up a problem also requires a scalable Machine Learning approach. This could be achieved by designing distributed algorithms or by devising special hardware like the neuro chips of Intel or the TPUs of Google. While there exist many different open-source frameworks for Machine Learning or Deep Learning like TensorFlow or PyTorch, there is currently no open-source framework which integrates all three components (Learning, Thinking, Acting) to provide a uniform base to create powerful intelligent systems. More work needs to be done here to unify the perspectives on AI.

There is a general consensus that AI needs to be explainable and make responsible decisions, especially in cases where safety is critical like in autonomous driving or healthcare. Current AI systems are always based on Deep Learning which are very hard to interpret and only work in a black box manner. In order to make AI widely acceptable, transparency and explainability will be a key property and a great challenge for research and industry, which requires more thoughts and innovation.

\section{How to prepare for the next AI wave?}

Right now, AI seems to be everywhere, and everyone who is lacking AI technologies seems to be out of place. But the right thing to do is not to adapt to the existing trend but to prepare for the next AI wave.

As more and more research will be done on AI, a first step to get into the community, other people, and companies is to attend international conferences about AI topics. Since AI is a very wide field, there exist many top venues like NeurIPS, ICML, ICLR, CVPR, AAAI, IJCAI, which are held annually with thousands of visitors all around the world. Visiting these conferences is a good way to start getting into the topics and trends.

Another important step is to create a social network to get in contact with experts and other companies to gain know-how and to collaborate on common projects. This can be initially done at such conferences.

The next crucial step to prepare for the next AI wave is to hire experts from required fields. This can be done after gaining the know-how about the own requirements and after extensive exchange with other experts and companies. Knowing about all of these is important to hire the right people for the right purpose and to maximize the effect within the own company.

For more information on this subject, please feel free to visit our AI-Lab at ( http://www.mobile.ifi.lmu.de/ai-lab/). We are looking forward to your visit!

Thomy Phan, Dr. Sebastian Feld, Prof. Dr. Claudia Linnhoff-Popien

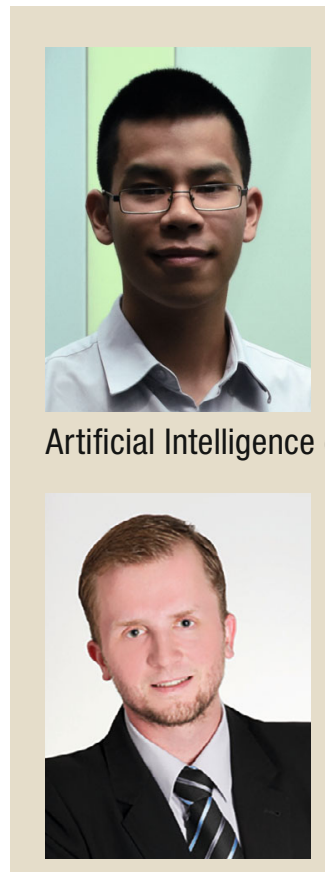

\section{Thomy Phan}

Thomy Phan is a PhD Student in Computer Science at LMU Munich. His research focuses on artificial intelligence and autonomous systems. He is head of the Al Laboratory of the Mobile and Distributed Systems Group at LMU Munich and member of the Organizing Committee of the First International Symposium on Applied (ISAAl'19).

\section{Dr. Sebastian Feld}

Dr. Sebastian Feld is head of the Quantum Applications and Research Lab (QAR-Lab) at the Mobile and Distributed Systems Group of the LMU Munich.

Currently, he pursues the goal of habilitation with a main focus being on optimization problems and the application of quantum technology.

He joined LMU in 2013 and earned his doctorate in 2018 working on planning of alternative routes, time series analysis and geospatial trajectories.

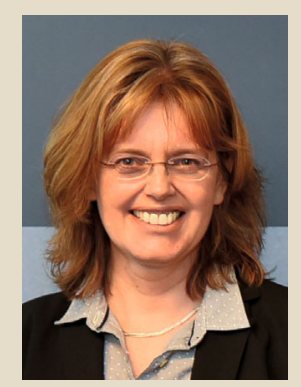

\section{Prof. Dr. Claudia Linnhoff-Popien}

Prof. Dr. Claudia Linnhoff-Popien holds the chair "Mobile and Distributed Systems" at the LMU Munich. She is board member of the Institute for Informatics, member of the "Münchner Kreis" and co-founder of the ALOQA GmbH. Further, she is head of the lead project "Innovationszentrum Mobiles Internet" of the Zentrum Digitalisierung.Bayern (ZD.B) funded by the state of Bavaria. She is also scientific advisor of the VIRALITY GmbH and chair of Digitale Stadt München e.V. 